\title{
A Protein-Based Three Terminal Electronic Device
}

\author{
ROSS RINALDI, GIUSEPPE MARUCCIO, ADRIANA BIASCO, \\ PAOLO VISCONTI, VALENTINA ARIMA, AND ROBERTO CINGOLANI \\ National Nanotechnology Laboratory of INFM, \\ Dipartimento di Ingegneria dell'Innovazione, University of Lecce, Lecce, Italy
}

\begin{abstract}
Because of their natural functional characteristics, involving interand intramolecular electron transfer, metalloproteins are good candidates for biomolecular nanoelectronics. In particular, blue copper proteins, such as azurin, can bind gold via a disulfide site present on its surface and they have a natural electron transfer activity that can be exploited for the realization of molecular switches whose conduction state can be controlled by tuning their redox state through an external voltage source. We report on the implementation of a prototype of protein transistor operating in air and in the solid state, based on this class of proteins. The three terminal devices exhibit various functions depending on the relative source-drain and gate-drain voltages bias, opening a way to the implementation of a new generation of logic architectures.
\end{abstract}

KEYWORDS: nanoscale devices; molecular electronics; bioelectronics; three terminal devices

\section{INTRODUCTION}

Nanobiotechnology has been boosted by an advancement in fabrication technologies that allows construction of artificial structures in the same range of biological entities. The fascinating world of the bio-self-assembly provides new opportunities and directions for future electronics, thus opening the way to a new generation of computational systems based on biomolecules and biostructures. Recent conceptstudies have demonstrated possible applications of bioelectronic devices to standard electronics (i.e., for rectification, amplification, and storage), as well as novel functionalities (i.e., for driving a biospecific assembly). ${ }^{1}$

The blue copper protein azurin, from Pseudomonas aeruginosa, is involved in bacterial respiratory phosphorylation. It has a molecular mass of $14,000 \mathrm{Da}$ and a distorted trigonal bipyramid structure for the copper active site, which plays the role of an electron reservoir in the electron transfer process. The functionality of this redox protein consists of performing electron transfer between other metalloproteins (cytochrome c551 and nitrite reductase) under physiologic conditions. ${ }^{2}$

The electron transfer mechanisms of azurin have been extensively studied, both experimentally by means of scanning tunnelling microscopy (STM) based studies and theoretically. ${ }^{3}$ Electrochemical in situ STM of adsorbate molecules with

Address for correspondence: Ross Rinaldi, National Nanotechnology Laboratory of INFM, Dipartimento di Ingegneria dell'Innovazione, University of Lecce, Lecce, Italy.

ross.rinaldi@unile.it 
low-lying redox levels strongly coupled to the environmental nuclear motion revealed that the STM process can be viewed as a coherent two-step electron transfer (ET) involving electron exchange between the local redox level and the manifolds of electronic levels in the substrate and tip. The notion of coherence implies that the intermediate electron or hole state after the first ET step does not fully relax vibrationally before the second ET step. ${ }^{4}$ Single-molecule behavior of redox metalloproteins, such as $P$. aeruginosa azurin, has been accurately and comprehensively investigated in liquid environment by single-molecule in situ STM. The properties of this class of molecules have pointed to ways of controlled orientation of welldefined monolayers of fully functional proteins, allowing a comprehensive characterization of the electronic properties of the immobilized molecules. 5,6

Recently Rinaldi et al. showed that it is possible to implement two terminal rectification devices based on an azurin monolayer. ${ }^{7,8}$ Taking into account that size downscaling by the usual top-down technologies is reaching its intrinsic limit, the development of new technologies for surface patterning based on self-assembly and nano-manipulation (i.e., using a bottom-up approach) becomes very important. In the case of the protein transistor we used a combination of these two approaches for the construction of the devices.

\section{RESULTS AND DISCUSSION}

The redox process involving the $\mathrm{Cu}$ active site of the protein in solutions is:

$$
\mathrm{Cu}^{2+}+\mathrm{e} \leftrightarrows \mathrm{Cu}^{+}
$$

This reversible reaction is activated by an electron transfer mechanism that continuously reconverts the copper oxidized state $\left(\mathrm{Cu}^{2+}\right)$ into the reduced states $\left(\mathrm{Cu}^{+}\right)$. Indeed the protein can exist in these two stable configurations, and its capability to work as a one-electron carrier depends on the equilibrium between these two states. The natural electron transfer activity can be exploited for the realization of molecular switches whose conduction state can be controlled by tuning their redox state through an external voltage source (gate).

The protein transistor was fabricated on $\mathrm{Si} / \mathrm{SiO}_{2}$ substrates, following the method reported elsewhere. ${ }^{7,8}$ The geometry of the device comprises two planar Au/Cr contacts separated by a gap in the range $50-100 \mathrm{~nm}$, which defines the gate width. An azurin monolayer was then immobilized onto the channel by means of surface functionalization and chemisorption. The formation of extended uniform protein monolayer was controlled by means of several surface techniques, as reported elsewhere. ${ }^{9}$ Moreover, a controlled protein orientation was obtained by optimizing the selfchemisorption process. ${ }^{7,8}$ Both commercial natural azurin and synthetic and purified azurin were used to implement the devices. A silver electrode was added on the back of the Si substrate as the gate, to control the source-drain conduction.

All the fabricated devices were tested at room temperature and ambient pressure. Prior to protein deposition, a negative control on the empty devices was performed to check the effective insulation between the source $(S)$, drain $(D)$, and gate $(G)$ terminals along the various current pathways. All these tests revealed $\mathrm{I}_{\mathrm{DS}}$ and $\mathrm{I}_{\mathrm{SG}}$ values smaller than $20 \mathrm{pA}$ and typical open circuit resistance larger than $100 \mathrm{G} \Omega$. 
In Figure $1 \mathrm{~A}$ we show the current voltage characteristics of a protein device for $V_{\mathrm{DS}}>0$ as a function of $V_{\mathrm{G}}$ in the range between $0 \mathrm{~V}$ and $4 \mathrm{~V}$ (first active region). As a general feature the $I_{\mathrm{DS}}$ current remains low (less than $20 \mathrm{pA}$ ) up to a $V_{\mathrm{DS}}$ bias voltage of about $2.3 \mathrm{~V}$ and then starts to increase, reaching $100 \mathrm{pA}$ intensity at $6 \mathrm{~V}$. A clear modulation effect is visible as a function of $V_{\mathrm{G}}$. The dependence of the $I_{\mathrm{DS}}$ current as a function of the $V_{\mathrm{G}}$ intensity is shown in FIGURE $2 \mathrm{~A}$ for a fixed value of $V_{\mathrm{DS}}$ $\left(V_{\mathrm{DS}}=4.5 \mathrm{~V}\right)$. The current increases up to a maximum value of $200 \mathrm{pA}$ at $V_{\mathrm{G}}=1.1 \mathrm{~V}$, then it decreases to the $100 \mathrm{pA}$ level at $V_{\mathrm{G}}=2 \mathrm{~V}$, and finally to the open circuit value at the higher applied gate voltages.

A similar set of measurements were made under positive and negative $V_{\mathrm{DS}}$ bias with positive (FIG.1 A and C, respectively) and negative (FIG. 1B and D, respectively) gate bias. Four different active region resulted: $V_{\mathrm{DS}}>0, V_{\mathrm{G}}>0$ (FIG. $1 \mathrm{~A}$ ); $V_{\mathrm{DS}}>0, V_{\mathrm{G}}<0$ (FIG. 1B); $V_{\mathrm{DS}}<0, V_{\mathrm{G}}>0$ (FIG. 1C); $V_{\mathrm{DS}}<0, V_{\mathrm{G}}<0$ (FIG. 1D). In FIGURE $1 \mathrm{~B}$, by decreasing the gate potential, the current increases to $160 \mathrm{pA}$ at $V_{\mathrm{DS}}$ $=6 \mathrm{~V}$. The corresponding dependence of the $I_{\mathrm{DS}}$ current as a function of $V_{\mathrm{G}}$ is shown in FIGURE $2 \mathrm{~B}$ for a $V_{\mathrm{DS}}$ value of $5 \mathrm{~V}$. In this case we did not observed a peaked dependence, as in the case of FIGURE $2 \mathrm{~A}$, but a smooth increase and saturation at $115 \mathrm{pA}$ in the range between -2.5 and $-4 \mathrm{~V}$. In the last two situations-that is, $V_{\mathrm{DS}}<0$, $V_{\mathrm{G}}>0$ (FIG. 2C) and $V_{\mathrm{DS}}<0, V_{\mathrm{G}}<0$ (FIG. 2D)-we observed a decrease and an increase (in absolute value) of the $I_{\mathrm{DS}}$ negative current with increasing gate voltage in the two cases, respectively. As a general trend, the current increases linearly for $V_{\mathrm{DS}}$ voltages greater than $3 \mathrm{~V}$ and then saturates in the range between 4.5 and $6 \mathrm{~V}$. In this voltage range the operation of the protein transistor resembles that of an inorganic MOS-FET in the saturation region, with constant $I_{\mathrm{DS}}$ current value for each curve.

To better understand the conduction mechanism in the protein FET and the origin of the different modulation effect in the four operating modes we need to summarize the Azurin electronic properties. As mentioned above, azurin is a blue-copper electron transfer protein. ${ }^{10}$ Many important chemical and biological processes involve long-range transfer of electronic excitation energy or electric charges between donor and acceptor molecules. At large separations (several angstroms), there is no direct donor-acceptor (DA) coupling, and intervening "bridge" sites that promote the transfer are required. This mechanism has been investigated in many biological molecules, such as cytochromes, hemoglobins, DNA, and docked proteins, on time scales down to the femtosecond range. It is generally found that, in the non adiabatic regime, the rate of electron transfer decays roughly exponentially with distance between donor and acceptor sites. One of the key questions in the study of acceptorbridge-donor complexes is the nature of the electron transfer process, which could be either a sequential (incoherent) hopping between adjacent sites or a direct quantum tunneling (superexchange) between the donor and acceptor. In the former case the bridge provides intermediate sites in which the excitation resides, whereas the only role of the bridge in the latter case is to provide virtual orbitals that determine the effective DA coupling. ${ }^{11,12}$ Usually, a unified description that includes sequential and tunneling processes is required to describe precisely the ET phenomena in biological media. For example, recent experiments performed on DNA to study the rate of charge transfer between two guanine bases indicate that there is a decrease in the transfer rate constant $K$ with increasing distance only if the guanine bases are 

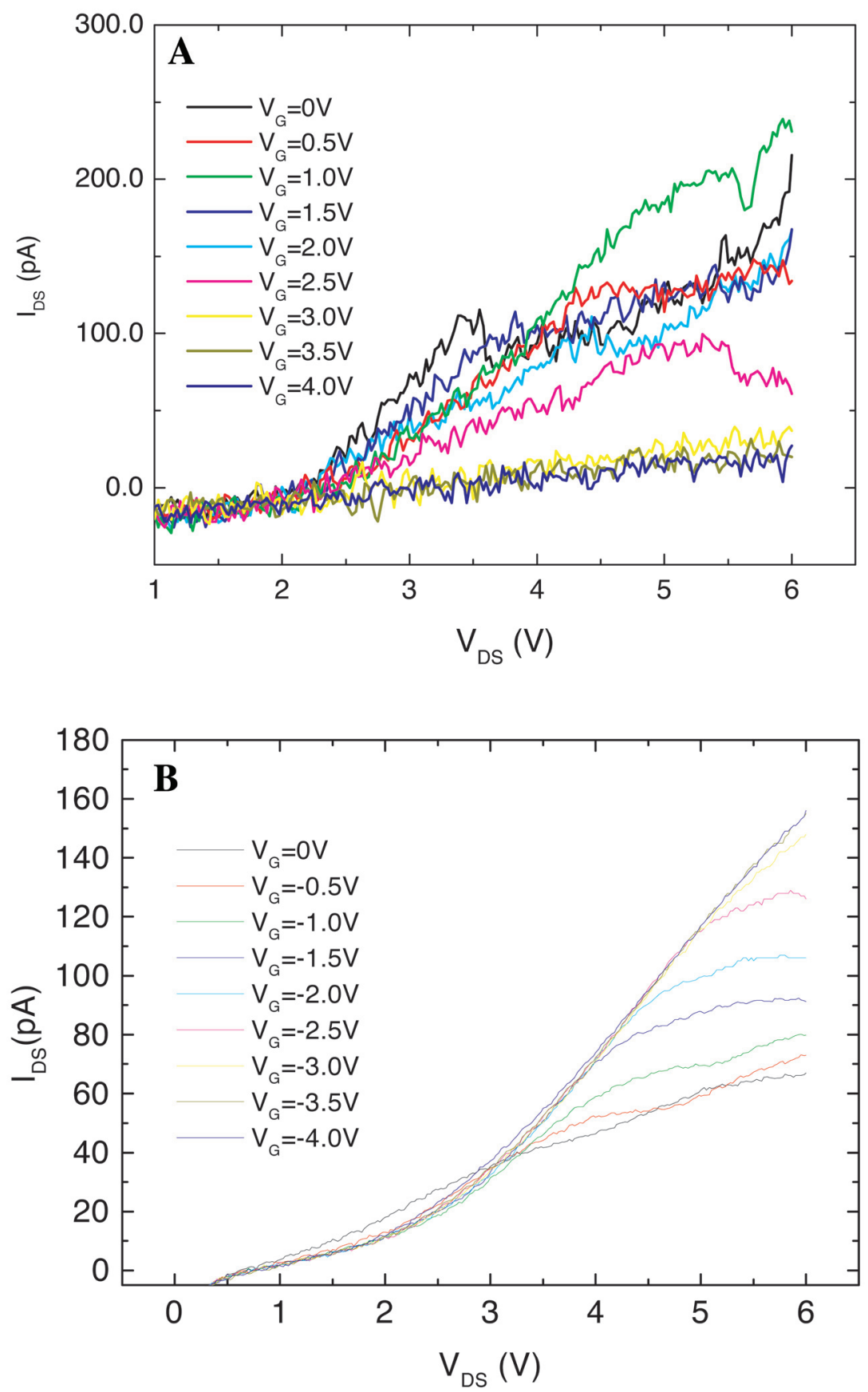

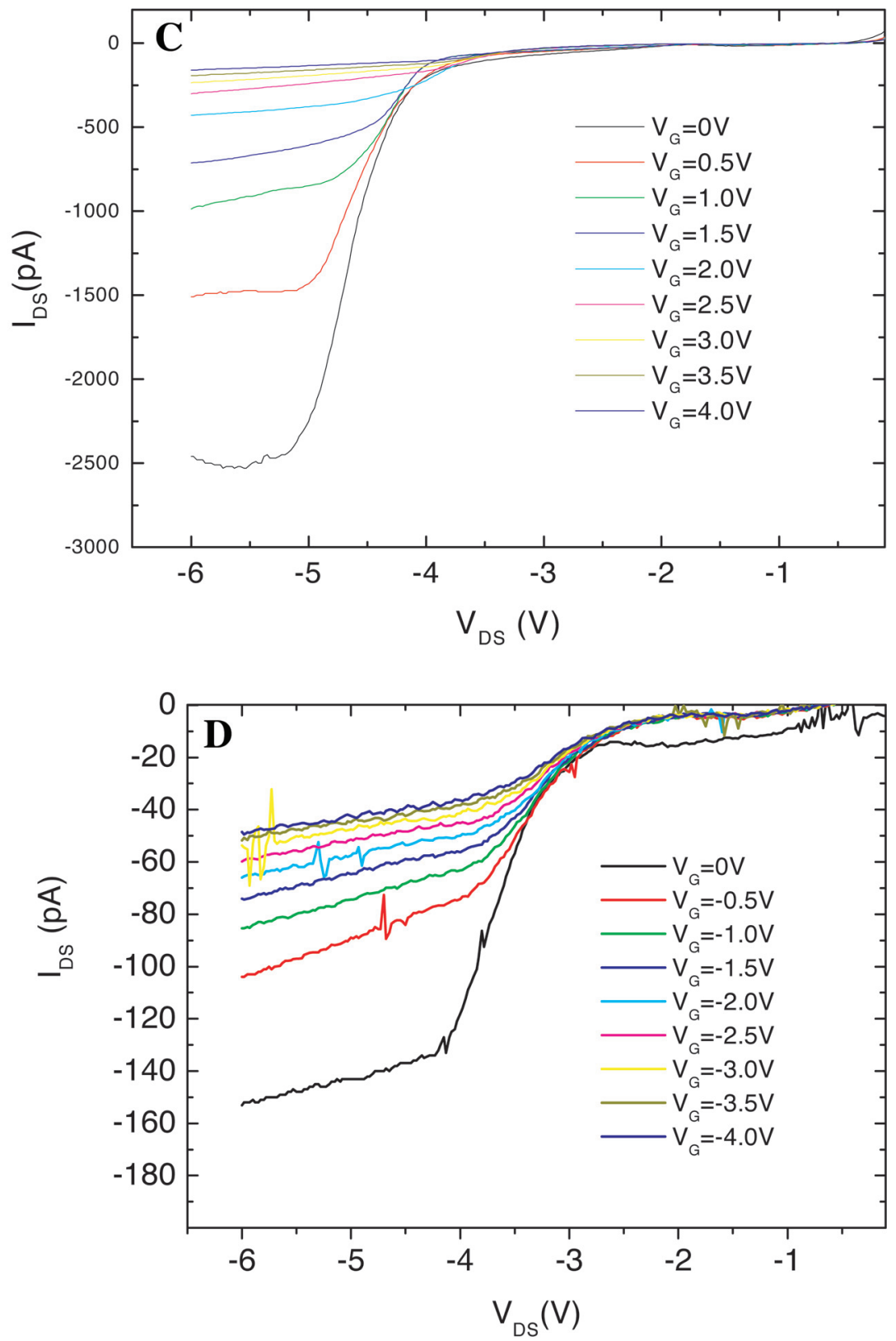

FIGURE 1. $I-V$ characteristics of the protein transistor in the four active regions: (A) $V_{\mathrm{DS}}>0, V_{\mathrm{G}}>0$; (B) $V_{\mathrm{DS}}>0, V_{\mathrm{G}}<0$; (C) $V_{\mathrm{DS}}<0, V_{\mathrm{G}}>0$; and (D) $V_{\mathrm{DS}}<0, V_{\mathrm{G}}<0$. 

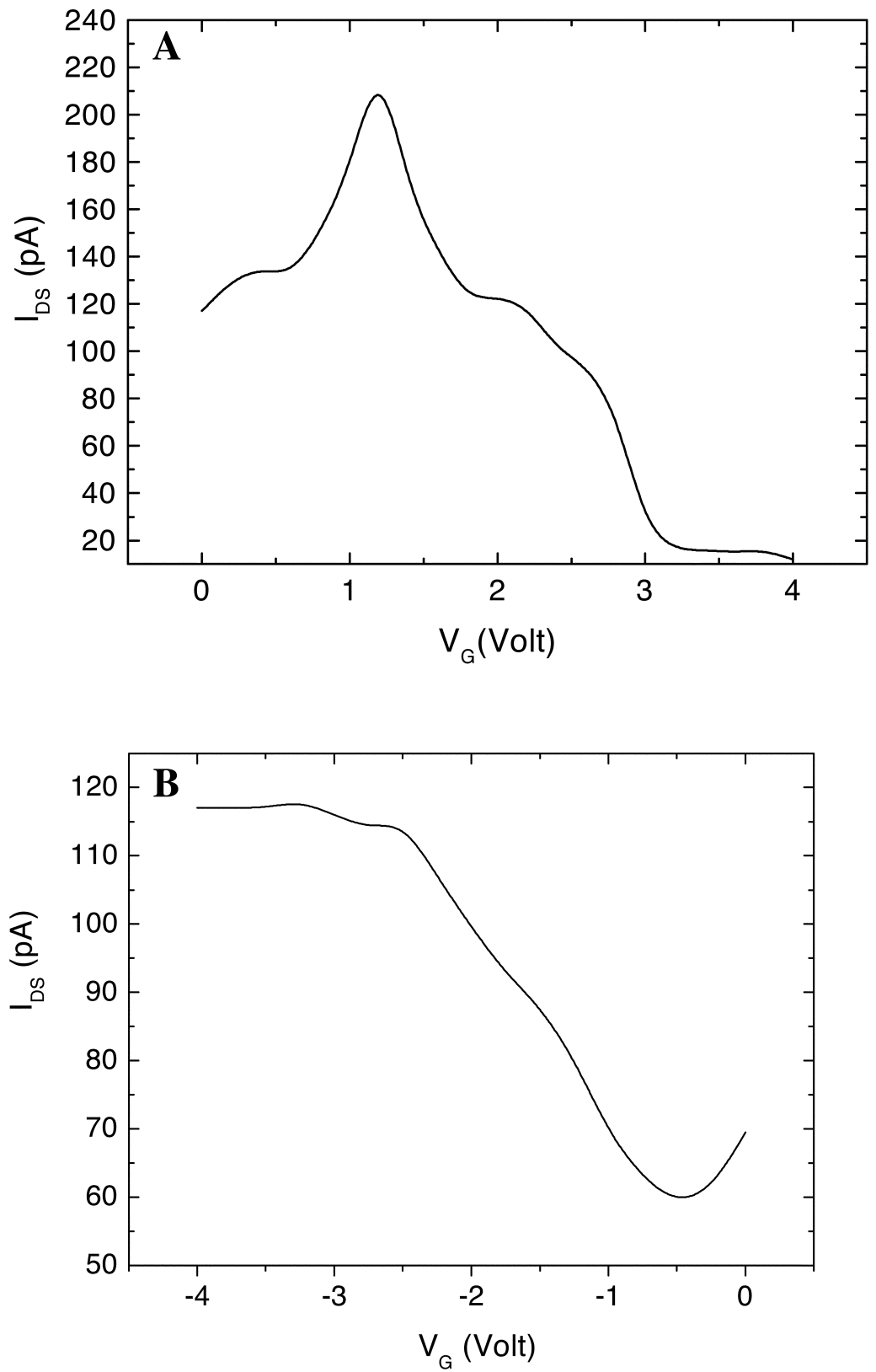

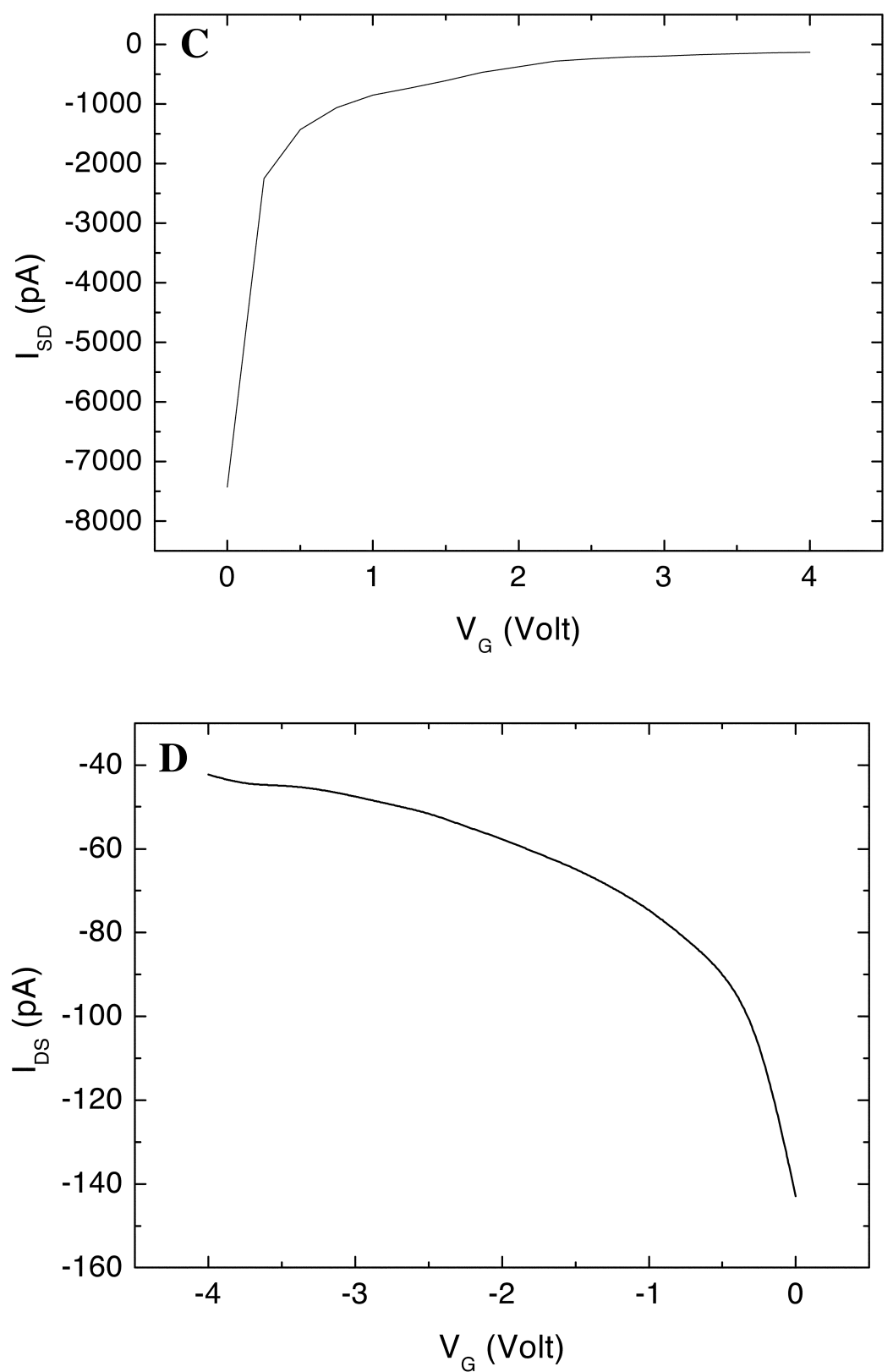

FIGURE 2. $I_{\mathrm{DS}}$ current intensities as a function of $V_{\mathrm{G}}$ at fixed $V_{\mathrm{DS}}$ values for the four active regions shown in FIGURE 1: (A) $V_{\mathrm{DS}}=4.5 \mathrm{~V}$; (B) $V_{\mathrm{DS}}=-5 \mathrm{~V}$; (C) $V_{\mathrm{DS}}=5 \mathrm{~V}$; and (D) $V_{\mathrm{DS}}=-5 \mathrm{~V}$. 
separated by no more than three base pairs; if more bridging base pairs are present, the transfer rates exhibit only a weak distance dependence. ${ }^{13}$ This finding indicates that a shift from coherent superexchange charge transfer (tunneling) at short distances to a process mediated by thermally induced hopping of charges at long distance can occur in biological media, without a visible reduction of the long range electron transfer effect.

Moreover, the effect of external perturbations, such as temperature change or a dissipative external bath, must be taken into account to properly describe the process that regulates the conduction properties of the materials. In some cases the occurrence of incoherent processes arising from inelastic scattering my result in the transformation from exponential decay to ohmic behavior, with the current dominated by inelastic processes and decreasing only slowly as the inverse of the distance between the donor and acceptor sites: ${ }^{14}$ this is analogous to the situation of electron transfer through tunneling barriers in semiconductor devices. Another important parameter in the evaluation of $K$ is the selection of the electron pathway inside the protein. ${ }^{15}$ In the case of an azurin monolayer deposited in the channel of our FET, both superexchange charge transfer inside the single azurin units and sequential hopping between adjacent proteins are involved in the conduction process. The existence of a strong internal dipole due to the surface charge distribution on the protein can affect the planar conduction in the gate channel depending whether the dipoles of the proteins in the layer are summed with a resultant in the same direction of the external source-drain field or not. ${ }^{7,8}$ This effect has been shown to be the origin of the rectification effects in planar two terminal devices, favoring conduction under a bias wing (positive or negative depending on the actual orientation of the proteins in the device). In the case of the characteristics of a device fabricated with commercial azurin, a similar effect is observed for positive gate voltages values (FIG. 1 A and C). In the case of the $I-V$ measurements in FIGURE $1 \mathrm{C}$, current intensities are higher than the corresponding values in FIGURE $1 \mathrm{~A}$ at least by a factor 10. For negative gate voltages the measured current intensities shown in FIGURE $1 \mathrm{~B}$ and D are similar.

The change in the gate voltage acts in various ways depending on the cross correlation of the signs of all the external applied voltages plus the effect of the internal dipole. As a general trend the $I_{\mathrm{DS}}$ current decreases with increasing absolute value of the applied $V_{\mathrm{G}}$. However, a different trend is usually observed in the case of $V_{\mathrm{DS}}>0, V_{\mathrm{G}}>0$ (FIG. 1A) where a peaked dependence was observed in most of the realized devices. The maximum of the current is always found about $V_{\mathrm{G}}=1 \mathrm{~V}$. This is a peculiar behavior of the protein device that is not found in any inorganic counterpart and could be of interest for the realization of electronic devices with novel functionalities. In the case of devices realized with synthetic and purified azurin the current increases by a factor 10 to 100 and the $V_{\mathrm{G}}$ resonance becomes sharper. The physical origin of this trend can be found in the effect of gate voltage on the molecular levels of the protein and, in turn, on the conduction pathways inside the molecule. The protein is chemisorbed onto the $\mathrm{SiO}_{2}$ surface by the formation of a thiolate onto the silanized surface. In this way the protein sits on the surface with the axis connecting the thiolate with the $\mathrm{Cu}$ ion almost perpendicular to the surface. The vertical field that propagates along this direction (gate field) modifies the electronic properties of the molecule and the oxidation state of the $\mathrm{Cu}$ ion, since it is strongly coupled to the thiolate by the ET pathway inside the protein. This resonance effect 

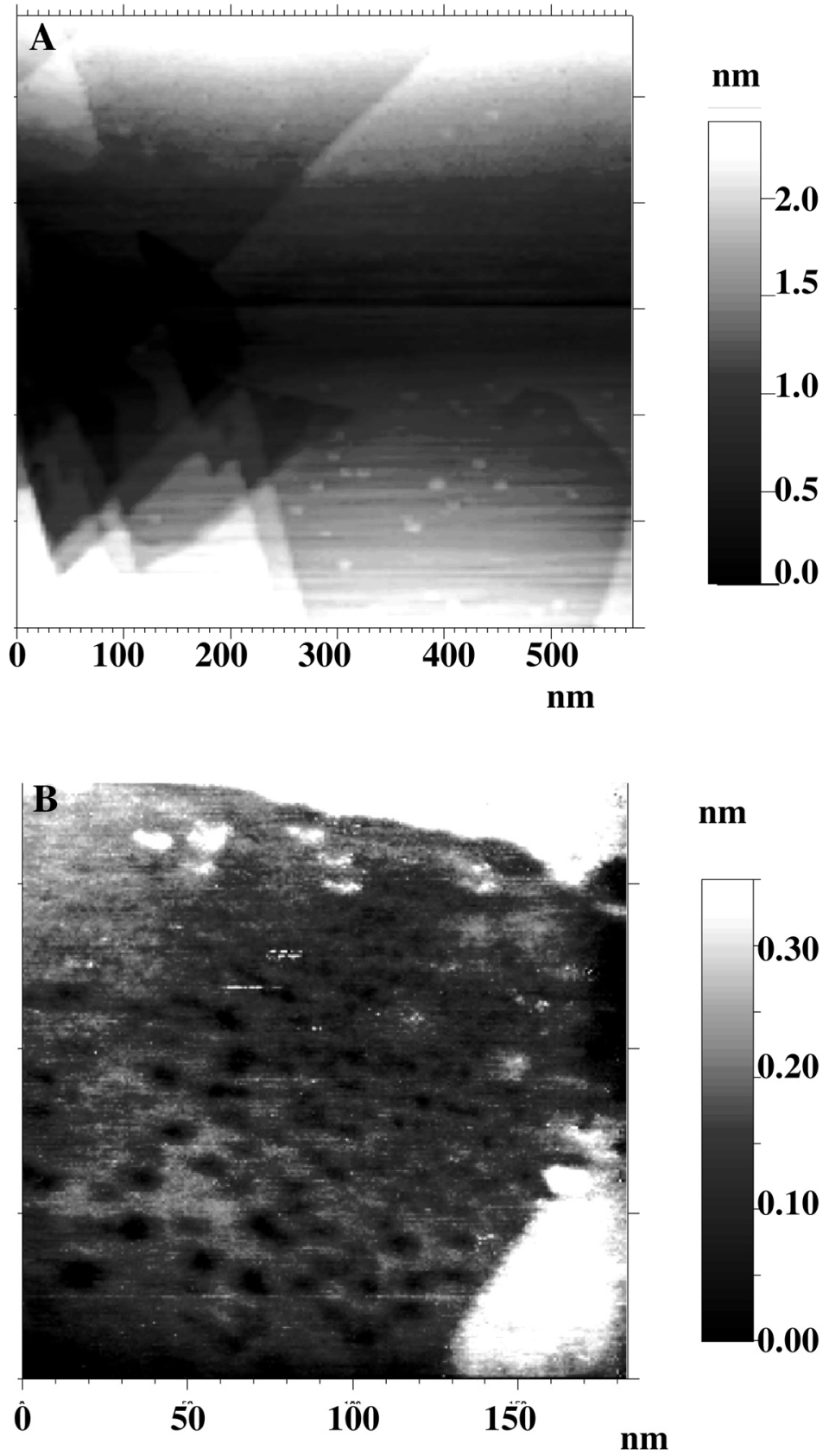

FIGURE 3. STM images of Azurin molecules chemisorbed on $\operatorname{Au}(111)$ substrates: (A) $I_{\text {tunn }}=0.5 \mathrm{nA}, V_{\text {gap }}=-1 \mathrm{~V}$ and (B) $I_{\text {tunn }}=0.5 \mathrm{nA}, V_{\text {gap }}=-0.5 \mathrm{~V}$. 
has been already observed in electrochemical STM experiments ${ }^{16}$ and in standard STM experiments performed on azurin molecules chemisorbed on $\mathrm{Au}(111)$ substrates at room temperature and ambient pressure. It has been demonstrated that the STM detection of the protein at the surface strongly depends on the applied bias between the tip and the gold substrate. FIGURE 3 shows two typical STM images recorded by keeping the tunneling current intensity constant to a value of $0.5 \mathrm{nA}$ and changing the gap voltage from $-1 \mathrm{~V}$ (FIG. 3 A) to $-0.5 \mathrm{~V}$ (FIG. 3B). Note that these measurements are carried out on azurin molecules immobilized in the solid state onto the gold surface; that is, in a condition very similar to that of the devices and totally different from that normally used in other experiments in solution. In the first image the proteins are visible on the gold surface: bright spots with the form of globular structures with dimensions of $4-6 \mathrm{~nm}$ in diameter are imaged by the STM. When the gap voltage is reduced to $-0.5 \mathrm{~V}$ no more proteins are imaged, instead, at their sites on the surface, small black dips appear, due to the artifact of the piezo movement that follows the tip looking for the tunneling current. In the first case the applied potential generated a favorable level alignment between the Au substrate the molecular levels of the protein and the tip, resulting in a resonant transfer of electron through the molecular levels. An off-resonance condition is reached by shifting the gap voltage to $0.5 \mathrm{~V}$, just as in the case of FIGURE $2 \mathrm{~A}$. For positive gap voltages it was not possible to achieve stable tunneling conditions. This means that the tunneling out of occupied states (HOMO levels) is the most favorable process.

\section{CONCLUSIONS}

We have demonstrated the first protein transistor working in air and at room temperature by using azurin metalloproteins. Various modulation effects were observed depending on the relative source-drain and gate-drain voltage bias polarity. This particular behavior was explained in terms of tuning the resonant transfer through the redox level of the molecule by means of the gate field. The fabrication of complementary devices on the same chip permits the construction of fully integrated circuits with a very small occupation area. Finally, the issues of device reproducibility, ageing, and lifetime must be addressed for molecular electronics. Among all the fabricated nanodevices, only a limited group exhibit a clear and reproducible gate effect, whereas in the others significant current fluctuations and failure occurred. Inspection by means of scanning electron microscope demonstrated that the failure was primarily due to the electrode burning and possibly metal electro-migration during operation. Electromagnetic field simulations are in progress, using finite element methods, to elucidate this important issue, to improve the device design, and, in turn, the device reproducibility.

\section{ACKNOWLEDGMENTS}

Support for this research was provided by the Italian Istituto Nazionale per la Fisica della Materia (PRA-SINPROT) and by the EU (SAMBA Project). 


\section{REFERENCES}

1. BASHIR, R. 2001. DNA-mediated artificial nanobiostructures: state of the art and future directions. Superlattices Microstructures 29(1): 1-16.

2. Adman, E.T. 1985. Structure and function of small blue copper proteins. In Topics in Molecular and Structural Biology, Vol. 1, Metalloproteins. P.M. Harrison, Ed: 1-42. Chemie Verlag, Weinheim.

3. Kuznetsov, A.M. \& J. Ulstrup. 1999. Electron Transfer in Chemistry and Biology. An Introduction to the Theory. Wiley, Chichester.

4. FriIs, E.P., Yu.I. Kharkats, A.M. KuZnetsov, et al. 1998. In situ scanning tunnelling microscopy of transition metal complexes and redox metalloproteins as a vibrationally coherent electronic three-level process. J. Phys. Chem. B 102: 7851-7859.

5. Zhang, J., Q. ChI, A.M. KuZnetsov, et al. 2002. Electronic properties of functional biomolecules at metal/aqueous solution interfaces. J. Phys. Chem. B 106(6): 1131-1152.

6. Contera, S.A. \& H. IwASAKI. 2002. Imaging the proteins pseudoazurin and apopseudoazurin on gold by STM in air: effect of the bias voltage. Ultramicroscopy 91: 231-243.

7. Rinaldi, R., A. Biasco, G. Maruccio, et al. 2002. Solid-state molecular rectifier based on self-organized metalloproteins. Advanced Materials. 20: 1453-1457.

8. Rinaldi, R., A. Biasco, G. Maruccio, et al. 2003. Electronic rectification in protein devices. Appl. Phys. Lett. 82: 472-474.

9. Schnyder, B., R. Kotz, D. Alliata \& P. Facci. 2002. Comparison of the self-chemisorption of azurin on gold and oxygen terminated surfaces. Surface Interface Anal. 34: 40.

10. Broo, A. \& S. Larsson. 1991. Electron-transfer in azurin and the role of aromatic side groups of the protein. J. Phys. Chem. 95: 4925-4928.

11. Kuhn, O., V. Rupasov \& S. Mukamel. 1996. Effective bridge spectral density for long-range biological energy and charge transfer. J. Phys. Chem. 104: 5821-5833.

12. Davis, W.B., M.R. WAsielewski \& M.A. RATNER. 1997. Electron transfer rates in bridged molecular systems: a phenomenological approach to relaxation. J. Phys. Chem. A 101: 6158-6164.

13. Giese, B., J. Amaudrut, A.K. Köhler, et al. 2001. Direct observation of hole transfer through DNA by hopping between adenine bases and by tunnelling. Nature 412: 318.

14. Neofotistos, G., R. Lake \& S. Datta. 1992. The non-equilibrium Green's function method applied to double barrier resonant tunneling diodes. Phys. Rev. B 43: 2442.

15. Kawatsu, T., T. Kakitani \& T. Yamato. 2000. A novel method for determining the electron tunneling pathway in protein. Inorgan. Chim. Acta 300-302: 862-868.

16. Facci, P., D. Alliata \& S. Cannistraro. 2001. Potential-induced resonant tunneling through a redox metalloprotein probed by electrochemical scanning probe microscopy. Ultramicroscopy 89(4): 291-298. 